\title{
Coordination between mid-term maintenance outage decisions and short-term security-constrained scheduling in smart distribution systems
}

\author{
M.A. Fotouhi Ghazvini, Hugo Morais, Zita Vale
}

\begin{abstract}
A B S T R A C T
Distribution systems are the first volunteers experiencing the benefits of smart grids. The smart grid concept impacts the internal legislation and standards in grid-connected and isolated distribution systems. Demand side management, the main feature of smart grids, acquires clear meaning in low voltage distribution systems. In these networks, various coordination procedures are required between domestic, commercial and industrial consumers, producers and the system operator. Obviously, the technical basis for bidirectional communication is the prerequisite of developing such a coordination procedure. The main coordination is required when the operator tries to dispatch the producers according to their own preferences without neglecting its inherent responsibility. Maintenance decisions are first determined by generating companies, and then the operator has to check and probably modify them for final approval. In this paper the generation scheduling from the viewpoint of a distribution system operator (DSO) is formulated. The traditional task of the DSO is securing network reliability and quality. The effectiveness of the proposed method is assessed by applying it to a 6-bus and 9-bus distribution system.
\end{abstract}

Keywords:

Coordination

Distribution system

Generation scheduling

Maintenance scheduling

Network security constraints

Smart grid

\section{Introduction}

The medium and low voltage distribution networks in existing power systems are mainly unidirectional from the viewpoint of data exchange between the system operator, producers and the consumers [1]. The penetration of technologies that support bidirectional communication infrastructure in distribution systems enables them to function in a smarter way with effective coordination between market participants. Smart grids should operate in a competitive environment with the involved players competing to achieve individual goals and cooperating in order to ensure common goals. These common goals mainly contain system issues, aiming at ensuring reliability and security levels and minimizing operation costs. Consequently, the agent that handles the supervisory role in smart grids is concerned about maintenance outage schedules, because they naturally deteriorate the grid reliability and increase the operation cost [2]. Electricity markets usually include a market operator that manages the financial transactions and the implementation of market rules and an independent system operator (ISO), which manages the network and all the involved constraints, in addition to sellers and buyers that negotiate in the market [3]. It carries out the duty of achieving a good solution enabling to attain common goals and to provide players with the conditions to attain their individual goals.
Therefore, it cannot manage the system individually ignoring the desired plans of other sections. Completing this duty requires coordination between the system operator, the transmission companies, the producers and the consumers.

A method based on game theory for maintenance scheduling in the layer of producers and in the transmission level is introduced in [4]. In this model producers decide their outage plans, considering their rivals' reactions. However, the outcome of this procedure is inapplicable due to the absence of supervision over security and reliability issues of the grid.

A coordinating mechanism based on incentives/disincentives among power producers and the ISO to achieve the final maintenance plan for a year ahead is introduced in [2]. This method expedites achieving a generation maintenance plan which satisfies the producer maximum profit objective while achieving a fair degree of reliability in each week of the year. In this model the coordination among producers and the ISO is provided through an iterative procedure. This iterative method requires several data exchange between the ISO and the producers, and also requires that each market participant solves its scheduling problem before submission. This is possible when we are talking about long-term planning; however, in short-term generation scheduling, which is usually a day or a week ahead, several data exchange requiring rescheduling at each destination is not possible.

In [5], the coordination between optimal maintenance scheduling of generating units and transmission lines in a vertically integrated power system is discussed. In this model the ISO runs the 


\section{Nomenclature}

Indices

$t \quad$ index of time periods (h)

$p \quad$ index of priority for maintenance plans offered from market participants to the DSO

$y \quad$ line index

$a, b \quad$ index for bus

$s \quad$ index of ESSs

$l \quad$ index of loads

$i \quad$ index of producers

$j \quad$ index of generating units

Variables

$x_{y}^{t} \quad$ binary decision variable for maintenance status of line $y$ at time period $t$ ( 0 if the line is on maintenance and 1 otherwise)

$x_{i j}^{t} \quad$ binary decision variable for maintenance status of generating unit $j$ of producer $i$ at time period $t$ ( 0 if the unit is on maintenance and 1 otherwise)

$z_{s}^{t} \quad$ binary decision variable for the discharging status of ESS

$s$ in period $t$ ( 1 if the unit is discharging and 0 otherwise)

$v_{s}^{t} \quad$ binary decision variable for the charging status of ESS $s$

in period $t$ ( 1 if the unit is charging and 0 otherwise)

$\alpha_{i j}(p) \quad$ binary decision variable for the acceptance of producer's $i$ th preferred plan for the outage plan of its generating unit $j$ ( 1 if it is accepted and 0 otherwise)

$\beta_{y}(p) \quad$ binary decision variable for the acceptance of $p$ th preferred plan for the outage plan of line $y$ ( 1 if it is accepted and 0 otherwise)

$u_{i j}^{t} \quad$ binary decision variable for online status of generating unit $j$ of producer $i$ in time period $t$ ( 1 if unit $j$ is on in period $t$ and 0 otherwise)

$d_{i j}^{t} \quad$ binary decision variable for shut-down status of generating unit $j$ belonging to producer $i$ in time period $t$ ( 1 if unit $j$ is shut down at the beginning of time period $t$ and 0 otherwise)

$s_{i j}^{t} \quad$ binary decision variable for start-up status of generating unit $j$ belonging to producer $i$ in time period $t$ ( 1 if unit $j$ starts up at the beginning of time period $t$ and 0 otherwise)

$Q_{i j}^{t} \quad$ reactive power generated by generating unit $j$ of producer $i$ in period $t$ (MVAR)

$P_{i j}^{t} \quad$ active power generated by generating unit $j$ of producer $i$ in period $t(\mathrm{MW})$

$P_{s}^{C}(t) \quad$ the power required for charging the ESS $s$ at time period $t$ (MW)

$P_{s}^{D}(t) \quad$ the discharging power of the ESS $s$ which injects into the grid at time period $t$ (MW)

$E_{s}^{\text {stored }}(t)$ energy storage level in ESS $s$ at the beginning of time period $t$ (MWh)

$\theta_{b}^{t} \quad$ the angle of bus $b$ at period $t$

$V_{b}^{t} \quad$ the voltage magnitude of bus $b$ at period $t$

$P_{b}^{g}(t) \quad$ active power injected to the grid by the generating units and the ESSs connected to bus $b$ at time period $t$ (MW)

$Q_{b}^{g}(t) \quad$ reactive power injected to the grid by all of the generating units connected to bus $b$ at time period $t$ (MVAR)

$P_{y}^{t} \quad$ the active power passing through line $y$ at time period $t$ (MW)
$Q_{y}^{t} \quad$ the reactive power passing through line $y$ at time period $t$ (MVAR)

$S_{y}^{t} \quad$ the apparent power that is passing through line $y$ (MVA)

Constants

$C_{i j}^{F}(t) \quad$ fixed cost of generating unit $j$ belonging to producer $i$ at each time period $(€)$

$C_{i j}^{P}(t) \quad$ production cost of generating unit $j$ belonging to pro-

ducer $i$ at each time period ( $€ /$ MWh)

$C_{i j}^{S} \quad$ start-up cost of generating unit $j$ of producer $i(€)$

$C_{s}^{c}(t) \quad$ the cost of charging the ESS $s$ at time period $t(€ / \mathrm{MWh})$

$J_{s}^{D}(t) \quad$ the grid income due to the participation of ESS $s$ in supplying energy during time period $t(€ / \mathrm{MWh})$

$n_{G} \quad$ number of producers

$n_{S} \quad$ number of ESSs

$n_{y} \quad$ number of lines

$n_{l} \quad$ number of loads

$n_{b} \quad$ number of buses

$T \quad$ total number of time periods (24 or 168 )

$P_{i j}^{M A X} \quad$ capacity of generating unit $j$ belonging to producer $i$ (MW)

$P_{i j}^{M I N} \quad$ minimum output power of generating unit $j$ belonging

MAX to producer $i$

$E_{s}^{M A X} \quad$ capacity of ESS $s(\mathrm{MWh})$

$E_{s}^{M I N} \quad$ minimum limit for the energy level of ESS $s$ (MWh)

$\eta_{s}^{C} \quad$ charging efficiency of ESS $s$

$\eta_{s}^{D} \quad$ discharging efficiency of ESS $s$

$C R_{S} \quad$ charge rate of ESS $s(\mathrm{MW} / \mathrm{h})$

$D R_{S} \quad$ discharge rate of ESS $s(\mathrm{MW} / \mathrm{h})$

$M R(t) \quad$ minimum reserve in period $t$ (MW)

$P_{b}^{d}(t) \quad$ active load demand and the discharge power of ESSs at bus $b$ and time period $t$ (MW)

$Q_{b}^{d}(t) \quad$ reactive load demand at bus $b$ and time period $t$ (MVAR)

$\theta_{b}^{\text {MAX }} \quad$ maximum bus angle for bus $b$

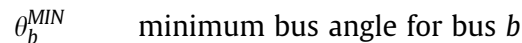

$V_{b}^{\text {MAX }} \quad$ maximum bus voltage magnitude for bus $b$

$V_{b}^{\text {MIN }} \quad$ minimum bus voltage magnitude for bus $b$

$S_{y}^{\text {MAX }} \quad$ maximum apparent power established at line $y$ (MVAR)

$W_{i j} \quad$ number of time periods required for the maintenance of generating unit $j$ belonging to producer $i$ in the specified time horizon

$\Psi_{y}^{t}(p) \quad$ the payment from transmission system owner to the DSO for each time period $(t)$ in order to increase the chance of acceptance of $p$ th preferred plan for the maintenance outage plan of line $y(€)$

$\Phi_{i j}^{t}(p) \quad$ the payment from producer $i$ to the DSO at each time period $(t)$ in order to increase the chance of acceptance of its $p$ th preferred plan for the outage plan of its generating unit $j(€)$

$G_{a b} \quad$ real term of the element $a, b$ in the bus admittance matrix maintenance scheduling problem considering the maintenance costs of market participants, and the final plan is compulsory for market players. A model similar to the approach introduced in
[5] is developed in [6]. The authors suggest considering the most preferred maintenance schedules of generation companies (GEN$\mathrm{CO}$ ) and of the transmission company (TRANSCO) when the 
short-term security-constrained unit commitment (SCUC) problem is being solved from the viewpoint of the ISO. It is assumed that the ISO does not carry the responsibility toward GENCOs and TRANSCOs to minimize their maintenance costs.

The ISO executes the SCUC in a restructured power system to plan a secure and economical hourly generation plan for the dayahead market. An efficient SCUC approach with AC constraints that obtains the minimum system operating cost while maintaining the security of power systems is introduced in [7]. The authors used the Benders decomposition to separate the unit commitment in the master problem from the network security check in sub -problems [7]. AC network security constraints, which include both transmission flow and bus voltage constraints, are checked to determine whether a converged and secure AC power flow is obtained.

Determining the optimal set of producers to be in service and participate in supplying the demand during each scheduling period (a day or a week) is an important issue in the daily operation and planning of power systems. In [8] an energy management model is proposed with the objective of minimizing the active power losses. In this model AC power flow equations plus the active and reactive power generation constraints impose limitations to the optimization problem. The concept of Virtual Power Producers (VPP), supervising agents for single and multiple isolated power systems, has been developed in [9], and its application on a real case study including various renewable resources has been examined.

There are different levels of smart grids available and calling a system smarter than the other system is getting popular. There is a wide range of technologies that can together form a smart grid; energy-related devices and interconnecting communication technologies are the two main categories of the involved technologies. Renewable energy resources, smart meters, energy storage systems (ESS), home area networks and many other technologies belong to this family.

In a smart environment, end-users have flexible offers in provision of energy and some other merits. Determining the structure rationally from numerous alternatives is the responsibility of the system operator. Lots of researches have been focused on this topic. A mixed-integer linear programming model is developed in [10] for the integrated plan and evaluation of distributed energy systems. This model minimizes overall energy cost for a test year by selecting the units to install and determining their operating schedules. Various renewable resources including solar, wind, biomass, as well as energy storage technologies are considered in the model [10]. In [11], an economic optimization model for the high level system design and unit commitment of a microgrid is developed. A mixed-integer linear programming model to maximize utilities profit and a linear programming model to minimize greenhouse gas emissions for a biomass based energy system is developed in [12].

The operation of a central controller for microgrids is described in [13]. The controller optimizes the operation of a microgrid operating under various market policies in the case of interconnection with upstream system [13].

Demand response is a good opportunity for consumers to participate in the smart grid environment, and represents significant benefits for the whole electricity market [14]. Demand response events affect the grid's security conditions, and therefore are managed by the DSO [15]. The DSO manages the distribution system and has the responsibility of voltage control. The methodology introduced in [14] considers the demand response to the electricity price variation imposed by the system operator in the presence of a reduction need, specifically designed for distribution systems. Demand response programs will have significant impact on existing power systems which about $20 \%$ of their capacity exists to meet the peak demand (i.e., about $5 \%$ of the time) [1]. However, imple- menting demand response programs avoids building extra capacity and consequently reduces the install cost in a power system.

The microgrid enters into the open market, buying and selling power to the grid, via an aggregator or similar energy service provider. The microgrid central controller maximizes the revenues of the aggregator by power exchange with the grid. The consumers also pay for their consumption at the open market prices [13].

The regulations in a smart distribution system should promote the application of smart grid supporting technologies for both the DSO and the network users [16]. In this paper, a scheme for effective and practical coordination between market members in a smart grid is proposed. The output of producers mid-term maintenance scheduling is submitted to the DSO and waits for its decision. The DSO is in charge of planning, operating and managing the distribution system and operates a monopoly businesses for its' regional network. In electricity market environment, DSOs seek the maximization of their income while ensuring the power quality and system reliability under strict network regulation. The way that the DSO reacts to suggested plans and the method it chooses for coordination are the main scopes of this research. This innovative strategy provides the operator with an efficient mechanism for short-term decision making considering the interests of the producers and the other players. This method is tested by a series of simulations on radial feeders, a 6 -bus distribution network $[17,18]$ is tested first and then the effectiveness of the proposed method is examined in a 9-bus system with higher penetration of distributed energy resources (DER).

\section{Smart distribution systems}

The traditional power systems are evolving into a new structure with greater levels of demand side management, and intensive use of distributed generation and ESSs closer to the consumption centers. The term "smart grid" is commonly used for futuristic distribution systems which will be more intelligent in comparison to conventional distribution systems [19]. The electricity distribution system as the first volunteer organization in power systems is experiencing a profound change, where blind and manual operations, along with the electromechanical components, are transforming into a "smart grid" [20]. The structure of distribution system is slightly changing due to the deregulation in power systems and government incentives for renewable resources. In smart distribution systems the energy flow is not just in one direction from the transformer substation to users as in passive systems. In this environment, customers have higher access to system conditions, which helps them to have better maintenance practices. To address the bidirectional flow patterns and online smart functions in real-time framework of daily operation in the distribution system infrastructure, major upgrades in communication structure are required $[20,21]$. These changes will have significant impact on the implementation of information technology in distribution systems which conventionally entail limited sensors and automation for data transfer.

Smart grids are often characterized in four layers: the distribution system with all physical elements, sensors and actuators, communication system, and finally the management system. Advanced distribution management system gives intelligence and smartness to the system and is known as the brain of future distribution systems [21].

In EU commission task force for smart grids [22], DSO is responsible for operating, maintaining, and developing the distribution system in the given area, where applicable, its interconnection with other systems. Moreover, the DSO is responsible for regional grid stability, integration of renewable sources at the distribution level and regional load balancing [22]. In future electricity markets, the responsibilities of the DSO resemble that of the transmission grid of today [22]. 
Deployment of the microgrids concept plays a leading role in establishing the evolution of conventional electrical grids toward smart grids. Microgrid is a low voltage distribution system comprising various DERs, controllable loads and ESSs and operates as a grid-connected system or as a controlled entity isolated from the main distribution system. An example for isolated microgrids is a low voltage regional network in a rural area $[13,23]$.

Planning in the smart grid framework can be divided into three different levels: synthesis optimization, design optimization and operation optimization. In the first two hierarchical process of planning, the components that appear in a system, their connections, technical characteristics of them and the substances that enter or exit each component are decided [10]. However, in this model the main purpose is achieving the optimal operating schedule in a system which has passed the previous two steps.

Renewable resources with intermittent nature are likely to be intensively used in most smart grids. These resources, due to their limited dispatchability, impose several challenges to the existing distribution infrastructure and to the ISO [20]. In many regions of the United States wind resources are considered as a must-take option for the ISO [20]. Variations in the production of renewable resources hamper the competition for the owners of these units when they participate in electricity markets [24]. The application of energy storage technologies which are under development partially alleviates the challenges that distribution systems face when trying to increase the share of renewable energy resources. The ability of storing considerable amounts of energy for several hours can provide the necessary flexibility for smoothing the output power of intermittent resources [24].

Implementation of smart grids requires a comprehensive regulatory framework in addition to technology, market and commercial consideration and information and communication technology. The emerging communication technologies will face the challenges of providing efficient power routing for higher cooperation between the members and enabling the consumers to manage their demand. The smart grid needs to enable its stakeholders to benefit from new ways of engaging with each other and performing information and energy exchange with each other in the market environment [1]. The purpose of the proposed model is to define a coordination procedure among many coordination procedures which might take place in a smart grid, and to propose a realistic methodology for maintenance scheduling in smart grids.

\section{Problem formulation and methodology}

In this section the formulation of the proposed method is discussed. As mentioned in the first section of this article, the main objective of the DSO in a smart distribution system is to determine a day-ahead or a week-ahead unit commitment to minimize the system operating cost while meeting the network security and the producers' constraints [7]. Similar methodologies and computing algorithms which are applied in transmission level, such as load flow and optimal power flow are needed in distribution system management [21].

In the centralized electricity framework, the system operator determines the maintenance plan that fulfills the requirement for the desired reliability level throughout the planning horizon while minimizing the operation cost of the system and imposes it to all producers [2].

When addressing the system planning problems in competitive environments, market participants' aims must be considered, specifically the schedule of their own equipment maintenance. The competitive nature of smart grids does not allow implementing a single stage maintenance scheduling structure used in centralized electricity networks. In such context, the producers that contribute in supplying the electrical energy to consumers should submit their mid-term maintenance outage plans to the system operator for approval. Similarly the main concern of the firms that take part in competitive markets, is obtaining the maximum benefit by increasing their profits. However, it should not be forgotten that the duties of the ISO which is in charge of maintaining the system security and guarantying an adequate level of reliability throughout the planning horizon cannot be overlooked.

In the proposed model, which is based on the idea that was first introduced in [6], the DSO considers the preference of producers and transmission lines for their maintenance outages. In the context of smart grids, the objective of the proposed method for forming a coordination between mid-term maintenance scheduling of generating units and transmission lines and short-term planning in the operator's level is to minimize operation costs over the scheduling period while satisfying operation and security constraints. The symbols that are used in the objective function and the constraints are introduced in the Nomenclature section. For the sake of simplicity, uncertainty in the system data is not considered, i.e. the demand data and wind farm generation forecasts are considered as known values. Forced outage rate of each generating unit properly illustrates the availability of each unit and depends on many factors mainly the previous maintenance experiences. This parameter should be considered as an important factor when generation scheduling is solved from the viewpoint of producers. In this model the effect of forced outage rate for each generating unit is included in the reserve constraint.

The DSO employs an approach to decide whether to permit, deny, or adjust planned outage schedules submitted by generating units and transmission lines in order to preserve the system security, while optimally committing, dispatching and allocating resources [25].

Each producer that might own several generating units solve the corresponding maintenance scheduling problem individually seeking their own profit maximization, considering the relevant constraints such as crew constraints [2]. The producers submit their maintenance outage plans to the DSO while assigning higher priorities to their preferred plans by offering payments to each time period of their desired maintenance window. This payment is in fact the penalty that producers can pay due to deteriorating the system reliability and security. The DSO has also accepted the risk of worsening the system reliability and security; therefore it might be questioned from the consumers or penalized by the higher supervisory organization. Each company suggests a set of plans with different priorities distinguished by the prices that they offer for each time period of outage in their preferred maintenance outage interval.

Several terms are included in the coordination objective function (1) of the DSO. The cost of providing energy for customers which is categorized as fixed costs, production costs and start-up costs of the producers is introduced in the objective function by the first three terms. The next two terms in the objective function relate to the charge cost and the discharge benefits for ESSs. The inclusion of producers' preference in operator's decisions is displayed in the last two terms.

$$
\operatorname{Min} \sum_{t=1}^{T}\left[\begin{array}{l}
\sum_{i=1}^{n_{G}} \sum_{j \in \Delta_{i}} C_{i j}^{F}(t) \cdot u_{i j}^{t} \cdot h(t)+\sum_{i=1}^{n_{G}} \sum_{j \in \Delta_{i}} C_{i j}^{P}(t) \cdot P_{i j}^{t} \cdot h(t)+\sum_{i=1}^{n_{G}} \sum_{j \in \Delta_{i}} C_{i j}^{S} \cdot S_{i j}^{t} \\
+\sum_{s=1}^{n_{S}} P_{s}^{C}(t) \cdot C_{s}^{C}(t) \cdot h(t)-\sum_{s=1}^{n_{s}} P_{s}^{D}(t) \cdot J_{s}^{D}(t) \cdot h(t) \\
-\sum_{i=1}^{n_{G}} \sum_{j \in \Delta_{i}} \sum_{p \in \Pi_{j}^{i}} \alpha_{i j}(p) \cdot \Phi_{i j}^{t}(p) \cdot\left(1-x_{i j}^{t}\right)-\sum_{y=1}^{n_{y}} \sum_{p \in \Pi_{y}} \beta_{y}(p) \cdot \Psi_{y}^{t}(p) \cdot\left(1-x_{y}^{t}\right)
\end{array}\right]
$$

Besides minimizing the total operation cost of the system, the preceding objective function maximizes the income of the system operator from the maintenance scheduling procedure which comes 


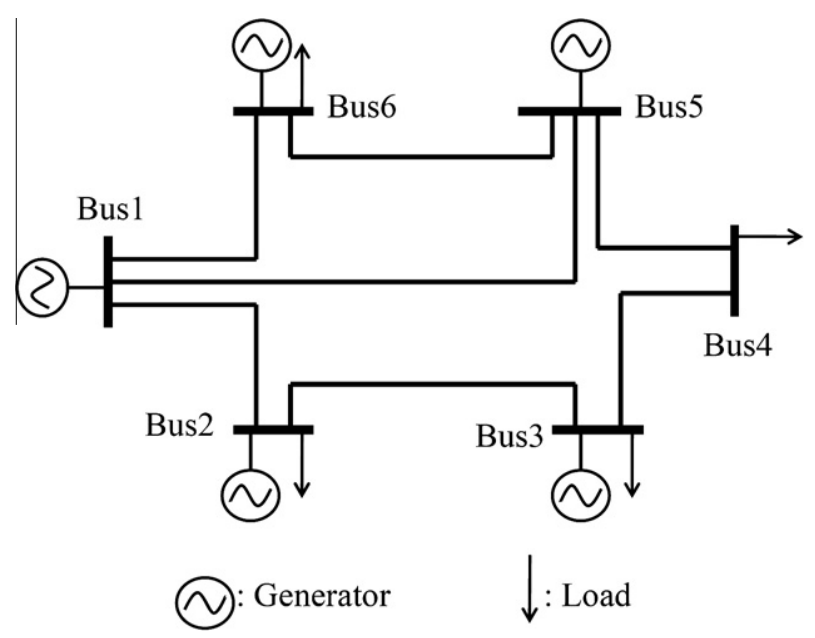

Fig. 1. One-line diagram for the 6-bus test system.

from its position as the regulatory section in smart grids. Additionally, the producers are also positive about this scheduling policy, because they can have their units in maintenance in their preferred maintenance windows, determined according to their own constraints and objectives.

The way that the forced outage rate involves in the formulation of the problem, depends on how the coordination procedure is defined and from which point of view the problem is seen. In this case, planning in the layer of producers is finished. Producers inform the DSO about the probability that each unit might not be available for service when required for the next scheduling time horizon. DSO ensures a net reserve above a particular threshold for all periods by considering constraint (2). Maintaining a specific reserve for each time period throughout the scheduling time span is one of the primary functions of the system operator [26].

$\sum_{i=1}^{n_{G}} \sum_{j \in \Delta_{i}} P_{i j}^{M A X} \cdot\left(1-x_{i j}^{t}\right) \cdot\left(1-F_{i j}\right)-\sum_{l=1}^{n_{l}} P_{l}^{D}(t) \geqslant M R(t), \quad \forall t$.

Constraint (3) ensures that each unit is maintained for the required number of time periods according to its submitted plan. Continuity between the time periods of maintenance is necessary; therefore, the operator adds this feature to the optimization problem when it considers constraint (4). Constraint (5) shows that a unit which is in maintenance cannot be committed. Constraints (6) and (7) enforce the logic of status change by defining the relationship between online, start-up and shut-down binary decision variables. Those units considered for maintenance in the following time horizon were not in maintenance during the last time period before the planning interval begins. However, the online state of the generating units during the last time period of the previous time horizon affects the start-up and shut-down status of the first
Table 2

Bus data.

\begin{tabular}{lll}
\hline Bus No. & Max-voltage $(\mathrm{pu})$ & Min-voltage $(\mathrm{pu})$ \\
\hline 1 & 1.05 & 0.95 \\
2 & 1.15 & 0.85 \\
3 & 1.15 & 0.85 \\
4 & 1.10 & 0.91 \\
5 & 1.01 & 0.97 \\
6 & 1.07 & 0.88 \\
\hline
\end{tabular}

time period. The value $u_{i j}^{0}$ shows the online state of each generating unit at this time period, and the planners need to know this value for each generating unit.

$\sum_{t=1}^{T}\left(1-x_{i j}^{t}\right)=W_{i j}, \quad \forall i, \forall j \in \Delta_{i}$

$x_{i j}^{t-1}-x_{i j}^{t} \leqslant\left(1-x_{i j}^{t+W_{i j}-1}\right) \quad \forall i, \forall j \in \Delta_{i}, t=2,3, \ldots, T$.

$u_{i j}^{t}+\left(1-x_{i j}^{t}\right) \leqslant 1, \quad \forall i, \forall j \in \Delta_{i}, \forall t$.

$s_{i j}^{t}-d_{i j}^{t}=u_{i j}^{t}-u_{i j}^{t-1} \quad \forall i, \forall j \in \Delta_{i}, \forall t$.

$s_{i j}^{t}+d_{i j}^{t} \leqslant 1 \quad \forall i, \forall j \in \Delta_{i}, \forall t$.

The active and reactive power that each online unit generates should be within a range, the following two constraints refer to this limitation of generating units.

$u_{i j}^{t} \cdot \stackrel{M I N}{P}_{i j}^{P} \leqslant P_{i j}^{t} \leqslant u_{i j}^{t} \cdot \stackrel{P_{i j}^{P A X}}{P} \quad \forall i, \forall j \in \Delta_{i}, \quad \forall t$.

$u_{i j}^{t} \cdot \stackrel{M I N}{Q}_{i j}^{M} \leqslant Q_{i j}^{t} \leqslant u_{i j}^{t} \cdot \stackrel{M A X}{Q}_{i j} \forall i, \forall j \in \Delta_{i}, \quad \forall t$.

Only one of the suggested outage plans might be selected by the DSO (10), (11).

$\sum_{p \in \Pi_{j}^{i}} \alpha_{i j}(p) \leqslant 1 \quad \forall i, \forall j \in \Delta_{i}$.

$\sum_{p \in \Pi_{y}} \beta_{y}(p) \leqslant 1 \quad \forall y$.

Eqs. (12) and (13) refer to active and reactive power flow equations [27]. The limits for bus voltage magnitude and bus voltage angle are shown in constraint (14) and (15) respectively. Eq. (16) shows the relation between active, reactive and apparent power passing through the lines. Constraint (17) refers to apparent power limit of the lines, or in other words, the line thermal limits.

$$
\begin{aligned}
P_{b}^{g}(t)-P_{b}^{d}(t)= & \sum_{a=1}^{n_{b}} V_{b}^{t} \cdot V_{a}^{t} \cdot\left(G_{b a} \cdot \cos \left(\theta_{b}^{t}-\theta_{a}^{t}\right)\right. \\
& \left.+B_{b a} \cdot \sin \left(\theta_{b}^{t}-\theta_{a}^{t}\right)\right) \quad \forall b, \forall t .
\end{aligned}
$$

Table 1

\begin{tabular}{|c|c|c|c|c|c|}
\hline Line No. & From bus & To bus & $Z_{\text {serial }}(\mathrm{pu})$ & $Y_{\text {shunt }}(\mathrm{pu})$ & Flow limit (MVA) \\
\hline 1 & 1 & 2 & $0.0038+j 0.509$ & $j 0.0012$ & 8.0 \\
\hline 2 & 2 & 3 & $0.0038+j 0.509$ & $j 0.0012$ & 7.0 \\
\hline 3 & 3 & 4 & $0.0038+j 0.509$ & $j 0.0012$ & 7.0 \\
\hline 4 & 4 & 5 & $0.0038+j 0.509$ & $j 0.0012$ & 8.0 \\
\hline 5 & 5 & 6 & $0.0038+j 0.509$ & $j 0.0012$ & 8.0 \\
\hline 6 & 6 & 1 & $0.2276+j 0.2961$ & $j 0.0025$ & 9.0 \\
\hline 7 & 1 & 5 & $0.2603+j 0.7382$ & $j 0.0008$ & 8.5 \\
\hline
\end{tabular}

Line data.

$S_{\text {base }}=100$ MVA, $V_{\text {base }}=25 \mathrm{kV}$. 


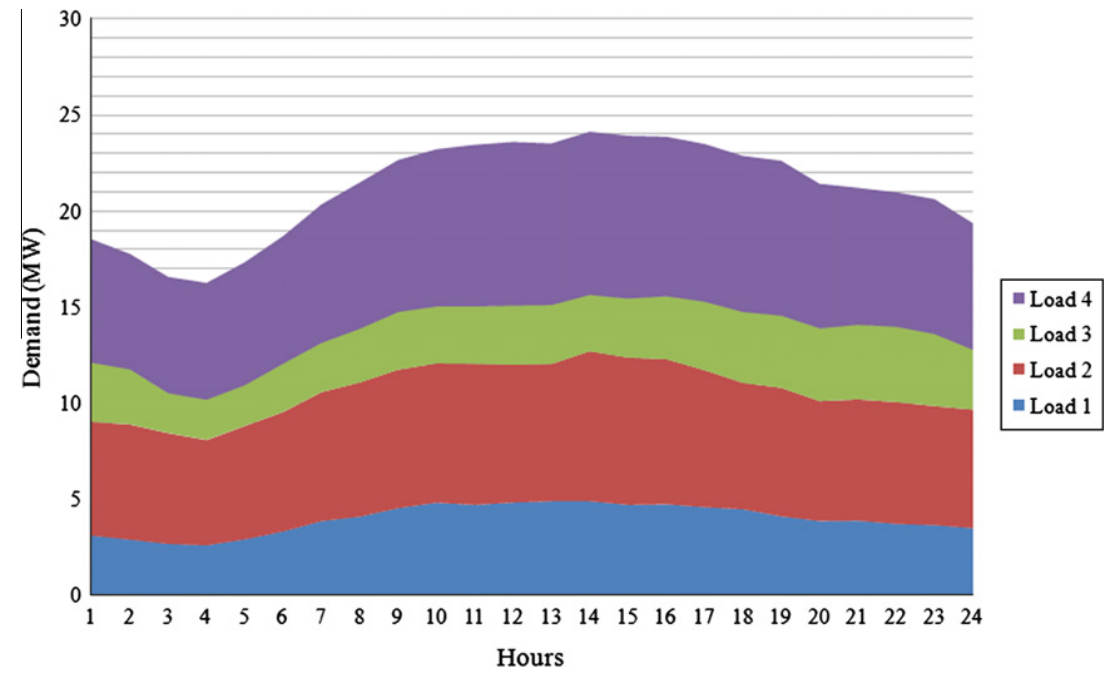

Fig. 2. Active demand for 6-bus test system. (For interpretation of the references to colour in this figure legend, the reader is referred to the web version of this article.)

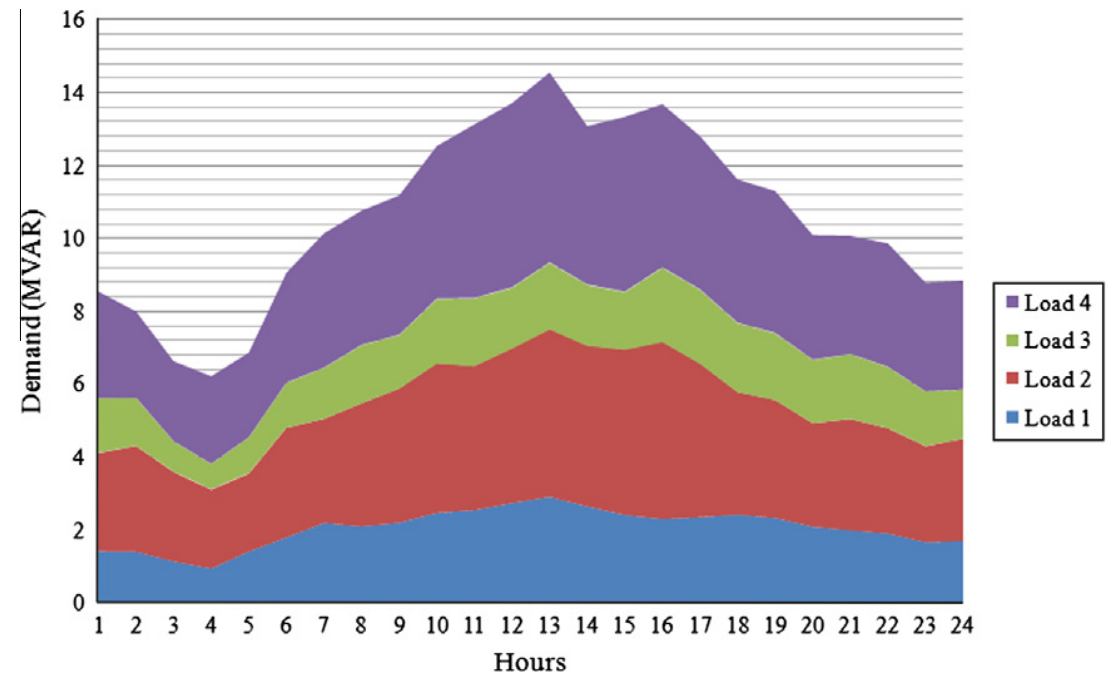

Fig. 3. Reactive demand for 6-bus test system. (For interpretation of the references to colour in this figure legend, the reader is referred to the web version of this article.)

Table 3

Generator technical data (case 1 ).

\begin{tabular}{|c|c|c|c|c|c|c|c|c|}
\hline Unit & Type & $\begin{array}{l}\text { Bus } \\
\text { No. }\end{array}$ & $\begin{array}{l}P_{\max } \\
(\mathrm{MW})\end{array}$ & $\begin{array}{l}P_{\min } \\
(\mathrm{MW})\end{array}$ & $\begin{array}{l}Q_{\max } \\
\text { (MVAR) }\end{array}$ & $\begin{array}{l}Q_{\min } \\
(M V A R)\end{array}$ & $\begin{array}{l}\text { Maintenance } \\
\text { duration (h) }\end{array}$ & $\begin{array}{l}\text { Forced } \\
\text { outage } \\
\text { rate }\end{array}$ \\
\hline G1 & $\begin{array}{l}\text { Hydro } \\
\text { power }\end{array}$ & 1 & 12 & 4 & 8 & 1 & 5 & 0.08 \\
\hline G2 & $\begin{array}{l}\text { Diesel } \\
\text { plant }\end{array}$ & 2 & 8 & 2 & 5 & 0.5 & 4 & 0.1 \\
\hline G3 & $\begin{array}{l}\text { Wind } \\
\text { farm }\end{array}$ & 3 & 9 & 4 & 4 & 0 & - & 0.05 \\
\hline G4 & $\begin{array}{l}\text { Biomass } \\
\text { plant }\end{array}$ & 5 & 8 & 3 & 5 & 1.5 & 8 & 0.07 \\
\hline G5 & Fuel cell & 6 & 3 & 1.5 & 1.5 & 0 & - & 0.01 \\
\hline
\end{tabular}

$Q_{b}^{g}(t)-Q_{b}^{d}(t)=\sum_{a=1}^{n_{b}} V_{b}^{t} \cdot V_{a}^{t} \cdot\left(G_{b a} \cdot \sin \left(\theta_{b}^{t}-\theta_{a}^{t}\right)\right.$

$\left.-B_{b a} \cdot \cos \left(\theta_{b}^{t}-\theta_{a}^{t}\right)\right) \quad \forall b, \forall t$.

$V_{b}^{\text {MIN }} \leqslant V_{b}^{t} \leqslant V_{b}^{\text {MAX }} \quad \forall b, \forall t$.

$\theta_{b}^{\text {MIN }} \leqslant \theta_{b}^{t} \leqslant \theta_{b}^{\text {MAX }} \quad \forall b, \forall t$.
Table 4

Generator cost data (case 1).

\begin{tabular}{llll}
\hline Unit & Fixed cost $(€ / \mathrm{h})$ & Production cost $(€ / \mathrm{MWh})$ & Start-up cost $(€)$ \\
\hline G1 & 61 & 37 & 98 \\
G2 & 72 & 159 & 111 \\
G3 & 63 & 32 & 18 \\
G4 & 72 & 52 & 103 \\
G5 & 85 & 182 & 76 \\
\hline
\end{tabular}

$\left(P_{y}^{t}\right)^{2}+\left(Q_{y}^{t}\right)^{2}=\left(S_{y}^{t}\right)^{2} \quad \forall y, \forall t$.

$S_{y}^{t} \leqslant S_{y}^{\text {MAX }} \quad \forall y, \forall t$.

The following constraint (18) indicates that the ESSs might only experience one of the charging or discharging conditions during each time period.

$z_{s}^{t}+v_{s}^{t} \leqslant 1 \quad \forall s, \forall t$.

The energy stored in ESS relates to its state during the previous time interval and the amount of storage at the start of that period (19). 
Table 5

Offered maintenance plans (case 1 ).

\begin{tabular}{clllllll}
\hline & Unit & $\begin{array}{l}\text { Priority } \\
1\end{array}$ & $\begin{array}{l}\text { Offered } \\
\text { price } \\
(€ / \mathrm{h})\end{array}$ & $\begin{array}{l}\text { Priority } \\
2\end{array}$ & $\begin{array}{l}\text { Offered } \\
\text { price } \\
(€ / \mathrm{h})\end{array}$ & $\begin{array}{l}\text { Priority } \\
3\end{array}$ & $\begin{array}{l}\text { Offered } \\
\text { cost } \\
(€ / \mathrm{h})\end{array}$ \\
\hline $\begin{array}{c}\text { Producer } \\
1\end{array}$ & G1 & $1-5$ & 40 & $4-8$ & 31 & $18-22$ & 17 \\
$\begin{array}{c}\text { Producer } \\
2\end{array}$ & G2 & $3-6$ & 52 & $21-24$ & 43 & $18-21$ & 11 \\
& G4 & $2-9$ & 63 & $5-12$ & 47 & $4-11$ & 31 \\
\hline
\end{tabular}

Table 6

Binary decision variables for online and start-up status.

\begin{tabular}{|c|c|c|c|c|c|c|c|c|c|c|}
\hline \multirow[t]{2}{*}{ Hours } & \multicolumn{5}{|c|}{ Online state } & \multicolumn{5}{|c|}{ Start-up state } \\
\hline & G1 & G2 & G3 & G4 & G5 & G1 & G2 & G3 & G4 & G5 \\
\hline 1 & 1 & 1 & 0 & 1 & 0 & 1 & 0 & 0 & 0 & 0 \\
\hline 2 & 1 & 0 & 0 & 1 & 0 & 0 & 0 & 0 & 0 & 0 \\
\hline 3 & 1 & 0 & 0 & 1 & 0 & 0 & 0 & 0 & 0 & 0 \\
\hline 4 & 1 & 0 & 1 & 0 & 0 & 0 & 0 & 1 & 0 & 0 \\
\hline 5 & 1 & 0 & 1 & 0 & 0 & 0 & 0 & 0 & 0 & 0 \\
\hline 6 & 1 & 0 & 1 & 0 & 0 & 0 & 0 & 0 & 0 & 0 \\
\hline 7 & 1 & 1 & 1 & 0 & 0 & 0 & 1 & 0 & 0 & 0 \\
\hline 8 & 1 & 1 & 1 & 0 & 0 & 0 & 0 & 0 & 0 & 0 \\
\hline 9 & 1 & 1 & 1 & 0 & 0 & 0 & 0 & 0 & 0 & 0 \\
\hline 10 & 1 & 1 & 1 & 0 & 0 & 0 & 0 & 0 & 0 & 0 \\
\hline 11 & 1 & 1 & 1 & 0 & 0 & 0 & 0 & 0 & 0 & 0 \\
\hline 12 & 1 & 0 & 1 & 1 & 0 & 0 & 0 & 0 & 1 & 0 \\
\hline 13 & 1 & 1 & 0 & 1 & 0 & 0 & 1 & 0 & 0 & 0 \\
\hline 14 & 1 & 1 & 0 & 1 & 0 & 0 & 0 & 0 & 0 & 0 \\
\hline 15 & 1 & 1 & 0 & 1 & 0 & 0 & 0 & 0 & 0 & 0 \\
\hline 16 & 1 & 1 & 0 & 1 & 0 & 0 & 0 & 0 & 0 & 0 \\
\hline 17 & 1 & 1 & 0 & 1 & 0 & 0 & 0 & 0 & 0 & 0 \\
\hline 18 & 0 & 1 & 1 & 1 & 1 & 0 & 0 & 1 & 0 & 1 \\
\hline 19 & 0 & 1 & 1 & 1 & 1 & 0 & 0 & 0 & 0 & 0 \\
\hline 20 & 0 & 1 & 1 & 1 & 1 & 0 & 0 & 0 & 0 & 0 \\
\hline 21 & 0 & 1 & 1 & 1 & 1 & 0 & 0 & 0 & 0 & 0 \\
\hline 22 & 0 & 1 & 1 & 1 & 1 & 0 & 0 & 0 & 0 & 0 \\
\hline 23 & 1 & 0 & 0 & 1 & 1 & 1 & 0 & 0 & 0 & 0 \\
\hline 24 & 1 & 0 & 0 & 1 & 0 & 0 & 0 & 0 & 0 & 0 \\
\hline
\end{tabular}

$$
\begin{aligned}
E_{s}^{\text {stored }}(t) & =E_{s}^{\text {stored }}(t-1)+h(t-1) \cdot\left(\eta_{s}^{C} \cdot P_{s}^{C}(t-1)\right. \\
& \left.-\frac{1}{\eta_{s}^{D}} P_{s}^{D}(t-1)\right) \quad \forall s, t=2,3, \ldots, T .
\end{aligned}
$$

Energy storage devices are defined by three parameters: storage capacity (MWh), charge rate (MW) and discharge rates (MW). In reality, the ESSs do not store all the received energy, and similarly provide less energy in comparison to the amount of energy depletion in the unit. These characteristics are modeled by charge efficiency and discharge efficiency. Constraints (20) and (21) model the limitation of ESSs related to their discharge rate and charge rate. The energy storage level in each ESS is limited by its capacity and the minimum threshold for energy level (22).

$\frac{1}{\eta_{s}^{D}} \cdot P_{s}^{D}(t) \leqslant D R_{s} \cdot z_{s}^{t} \cdot h(t) \quad \forall s, \forall t$.

$\eta_{s}^{C} \cdot P_{s}^{C}(t) \leqslant C R_{s} \cdot v_{s}^{t} \cdot h(t) \quad \forall s, \forall t$

$E_{s}^{M I N} \leqslant E_{s}^{\text {stored }}(t) \leqslant E_{s}^{M A X} \quad \forall s, \forall t$.

This problem is formulated as mixed-integer nonlinear programming problems that can be solved using commercially available software [28].

\section{Numerical results}

In this section we analyze two case studies, a 6-bus test system and its extension to a larger system with higher share for ESSs and renewable resources, to examine the performance and the effectiveness of the coordination procedure.

\subsection{Case 1}

Fig. 1 shows the one-line diagram of the 25-kV 6-bus distribution test system introduced in [18]. This test system includes five generating units and seven lines. Table 1 displays the line data, and gives the parameters such as lines' serial impedance and the value of the shunt capacitance which is defined in the line model. Table 2 refers to the limitations of voltage magnitude for each line. The bus angle is considered as a variable changing between $-\pi$ and $\pi$.

Loads 1-4 are respectively connected to buses $2,3,4$ and 6 . Fig. 2 shows the active power demand variation of the loads during the day on an hourly basis. Similarly the reactive power consumption of the four loads is depicted in Fig. 3. The time horizon of the study is $24 \mathrm{~h}$, and the data tables are given in this frame.

Two main producers own the generating units; producer 1 owns units 1 and 3 and the rest of generating units belong to producer 2 . Table 3 shows the technical data of generating units, and Table 4 gives the generators' cost data.

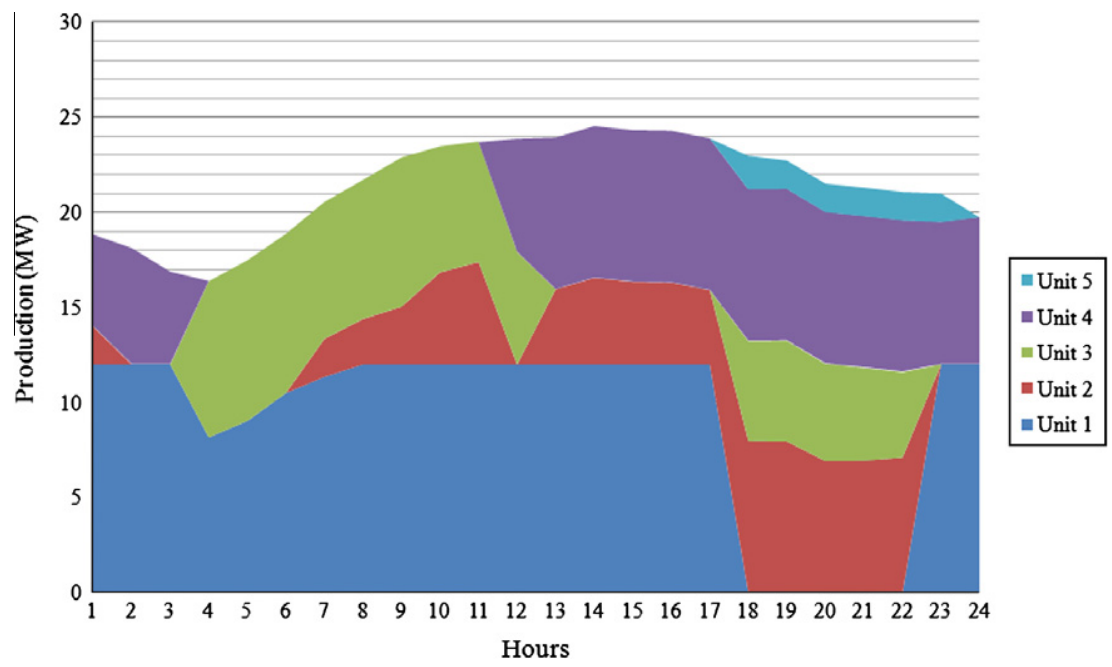

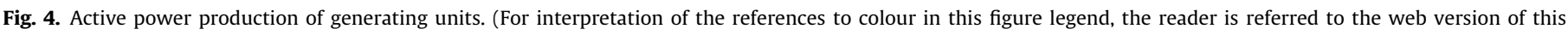
article.) 


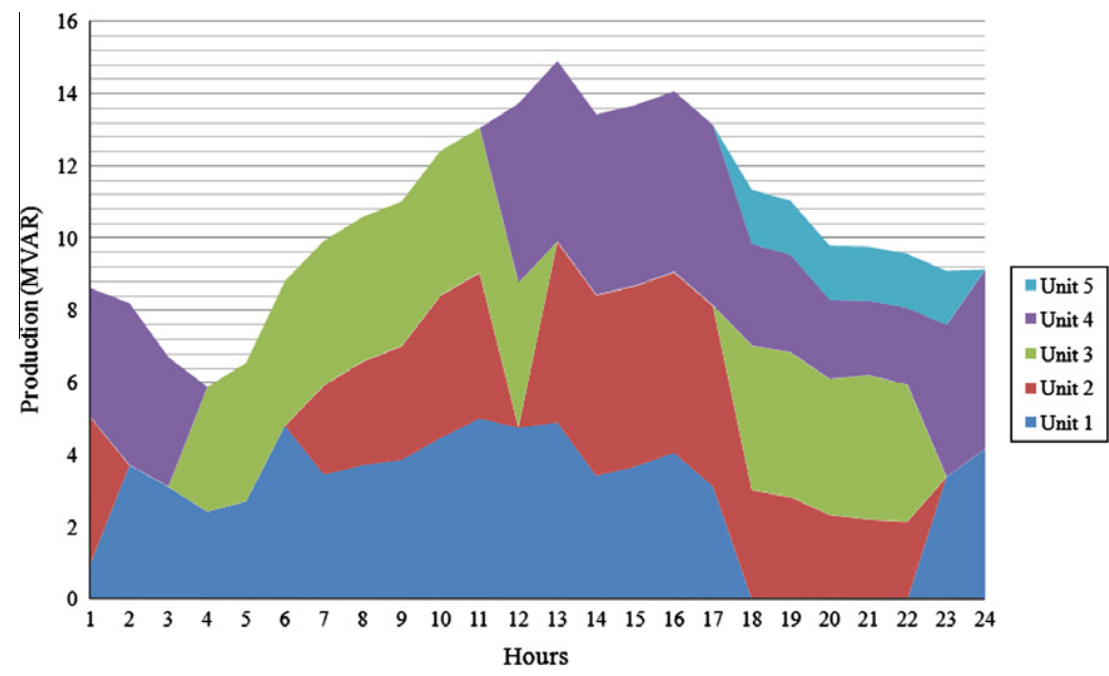

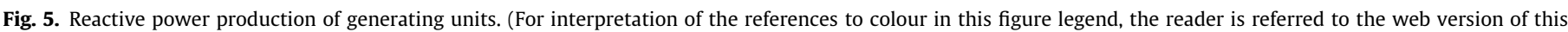
article.)

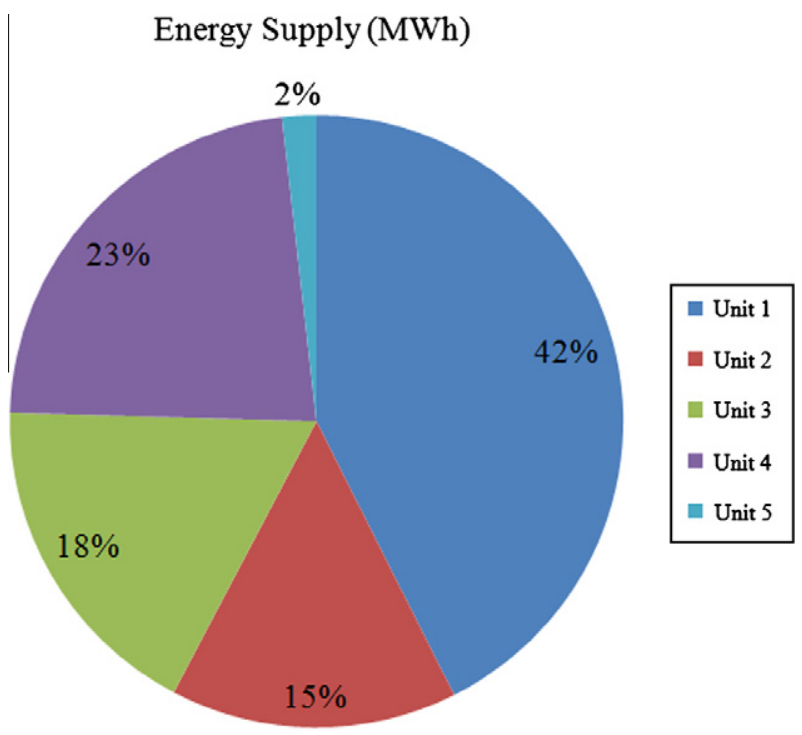

Fig. 6. Share of each generating unit to supply demand ( $24 \mathrm{~h}$ ). (For interpretation of the references to colour in this figure legend, the reader is referred to the web version of this article.)

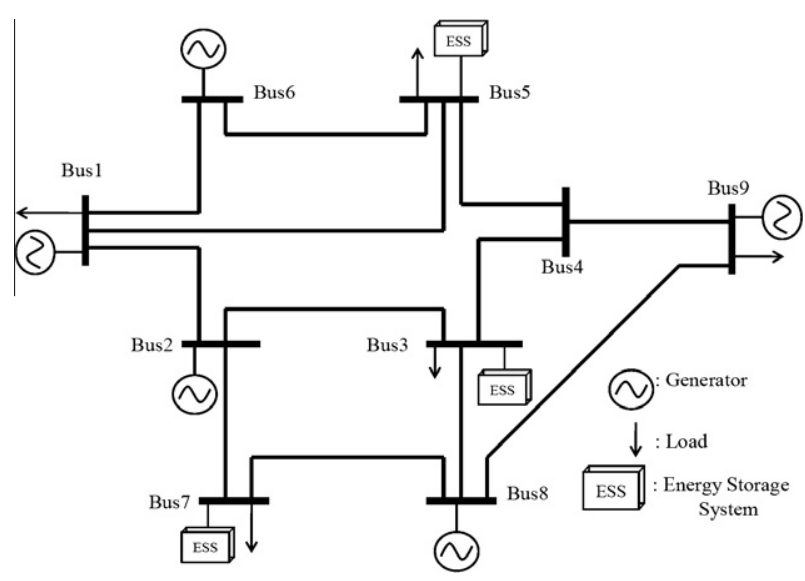

Fig. 7. One-line diagram for the 9-bus test system.
The effective supervision of the DSO is required to control and analyze the maintenance plans submitted by the producers. This is also an opportunity for the operator to reduce the cost by considering the payments that generating units propose for their preferred plans.

The two producers demonstrate their willingness to have the generating units that they own in maintenance during their preferred time periods. Table 5 shows their offered plans and their corresponding priority levels which are distinguished by the price that they suggest for each hour.

The DSO receives these suggestions and has the authority to modify, deny or accept them. From the viewpoint of the DSO, the minimum operation cost considering the payments from generation companies from the viewpoint of the DSO is 36,096 Euros. The final maintenance plan which is mandatory for the market participants for the day ahead agrees with the third priority of unit 1 for maintenance, first priority of unit 2 and last priority of unit 4 for its maintenance. The total payment to the DSO in the coordination procedure during the scheduling time horizon of one day is 541 Euros.

The dispatch variables for this case during the $24 \mathrm{~h}$ of planning are shown in Table 6. Figs. 4 and 5 refer to the contribution of each unit in satisfying the active and reactive load demand. Wind units are not dispatchable; therefore, the production forecast of the wind unit in our test system is considered as known data. Inherited binary variables for the last time period of the previous scheduling period affect the results and are considered in the simulation. It is also worth noting that the difference between the sum of units' production and the demand in active and reactive figures is due to the line loss which is included in our model by considering the serial impedance and the line shunt capacitance.

In Fig. 6 the share of each generating in supplying the energy needs of the smart grid during the considered $24 \mathrm{~h}$ is displayed.

Single stage maintenance scheduling is common in conventional power systems, and the final plan is appointed as a fixed schedule by the operator. For this case study the proposed approach can provide about $17 \%$ of the active demand at each hour as the system reserve for that period. However, the conventional one stage maintenance scheduling can ensure more than $22 \%$ of the active demand at each hour as the system reserve. One of the main responsibilities of the DSO is to maintain the reliability of the system at an acceptable level. Applying the proposed approach in comparison to the conventional maintenance scheduling 


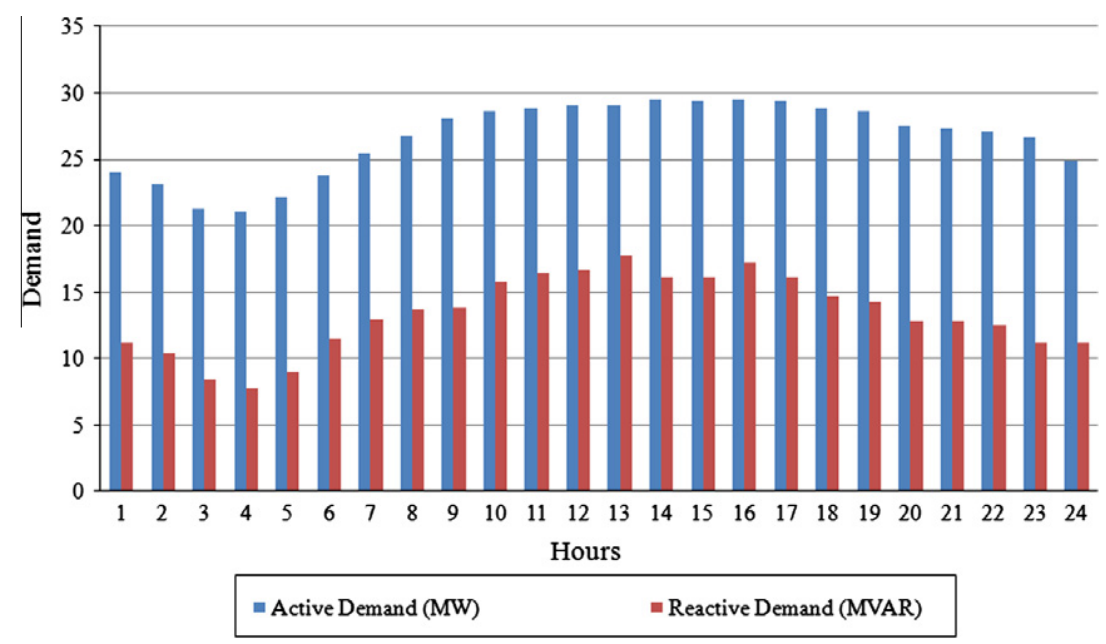

Fig. 8. System demand (case 2). (For interpretation of the references to colour in this figure legend, the reader is referred to the web version of this article.)

Table 7

Generator technical data (case 2).

\begin{tabular}{|c|c|c|c|c|c|c|c|c|}
\hline Unit & Type & $\begin{array}{l}\text { Bus } \\
\text { No. }\end{array}$ & $\begin{array}{l}P_{\max } \\
(\mathrm{MW})\end{array}$ & $\begin{array}{l}P_{\min } \\
(\mathrm{MW})\end{array}$ & $\begin{array}{l}Q_{\max } \\
(\mathrm{MVAR})\end{array}$ & $\begin{array}{l}Q_{\min } \\
\text { (MVAR) }\end{array}$ & $\begin{array}{l}\text { Maintenance } \\
\text { duration (h) }\end{array}$ & $\begin{array}{l}\text { Forced } \\
\text { outage } \\
\text { rate }\end{array}$ \\
\hline G1 & Fuel cell & 6 & 8 & 2 & 5.5 & 1 & - & 0.01 \\
\hline G2 & Hydro & 1 & 10 & 3 & 6 & 2 & 13 & 0.11 \\
\hline G3 & Biomass & 2 & 9.5 & 3 & 5 & 0.5 & 7 & 0.015 \\
\hline G4 & Solar & 9 & 9 & 5 & 7.5 & 1 & - & 0.01 \\
\hline G5 & Diesel & 8 & 11 & 1 & 7 & 2 & - & 0.09 \\
\hline
\end{tabular}

Table 8

Generator cost data (case 2).

\begin{tabular}{llll}
\hline Unit & Fixed cost $(€ / \mathrm{h})$ & Production cost $(€ / \mathrm{MWh})$ & Start-up cost $(€)$ \\
\hline G1 & 73 & 101 & 72 \\
G2 & 54 & 41 & 97 \\
G3 & 42 & 56 & 94 \\
G4 & 23 & 29 & 18 \\
G5 & 77 & 136 & 117 \\
\hline
\end{tabular}

represents additional revenue for the DSO. The DSO has accepted the risk of reducing the system reserve and consequently the reliability deterioration in exchange for the excess income. This revenue can be shared with system loads by means of reliability dependant demand response programs. In fact, these are alternative ways to remunerate these loads acceptance of a lowering in their energy supply quality level.

\subsection{Case 2}

The 6-bus test system introduced in case 1 has been extended to match to the characteristics of smart grids. The 9-bus test system as shown in Fig. 7 has 12 lines with 5 generating units. The to- tal active and reactive demand during the $24 \mathrm{~h}$ of planning is shown in Fig. 8.

The generating units' technical and cost data are shown in Tables 7 and 8 . The time horizon of the operation planning is $24 \mathrm{~h}$. In order to show the non-dispatchability feature of the solar unit, this has been considered offline during hours 1-5 and 21-24. Three ESSs are located in this system. The technical characteristics of these units are shown in Table 9.

The maintenance plans with different priorities are submitted by the generating units. In this case three priority levels are considered for units 2 and 3 . Unit 2 pays $51 € /$ hour if its suggested plan for maintenance during hours 12-24 achieves the acceptance, and $33 €$ /hour for hours 5-17. Unit 2 does not have the third priority and this means that if its first two desired plans are not approved, then it does not mind about other maintenance windows determined by the DSO. Unit 3 submits all the three plans allowed for each generating unit with their priority levels and the price that it will pay if it is considered for outage during those periods. It pays 45,32 and $26 €$ /hour respectively for hours $14-20,12-18$ and 612.

The model is implemented using DICOPT under GAMS [29] on a computer equipped with two Xeon X5450 processors, each one with 4 cores, clocking at $3.0 \mathrm{GHz}$ with $4 \mathrm{~GB}$ of RAM. The time required to attain the solution is $8.112 \mathrm{~s}$. The results show that none of the proposed maintenance windows by unit 2 are accepted. The DSO decides hours 1-13 for maintenance of unit 2 which does not fit to any of the plans proposed by this unit. The most preferred plan for unit 3 gains the acceptance. The total operation cost of the distribution system is $48251 €$. Unit 3 has to pay $315 €$ in order to benefit from the coordination procedure and to be in maintenance during its preferred time interval.

When intermittent resources receive higher share in smart grids, the benefits of ESSs can be easily understood. In this case study, the ESSs charge and discharge many times as shown in Fig. 9. During the hours that the system might experience shortage

Table 9

ESSs' technical and cost data.

\begin{tabular}{|c|c|c|c|c|c|c|c|c|}
\hline ESSs & Bus & Capacity (MWh) & $\begin{array}{l}\text { Charge rate } \\
(\mathrm{kW} / \mathrm{h})\end{array}$ & $\begin{array}{l}\text { Discharge rate } \\
(\mathrm{kW} / \mathrm{h})\end{array}$ & Charging efficiency & Discharging efficiency & $\begin{array}{l}\text { Charging cost } \\
(€ / M W h)\end{array}$ & $\begin{array}{l}\text { Discharging profit } \\
(€ / \mathrm{MWh})\end{array}$ \\
\hline U1 & 7 & 2 & 630 & 510 & 0.92 & 0.89 & 14 & 69 \\
\hline $\mathrm{U} 2$ & 5 & 3 & 220 & 270 & 0.83 & 0.82 & 21 & 83 \\
\hline U3 & 3 & 2 & 530 & 157 & 0.93 & 0.94 & 24 & 81 \\
\hline
\end{tabular}




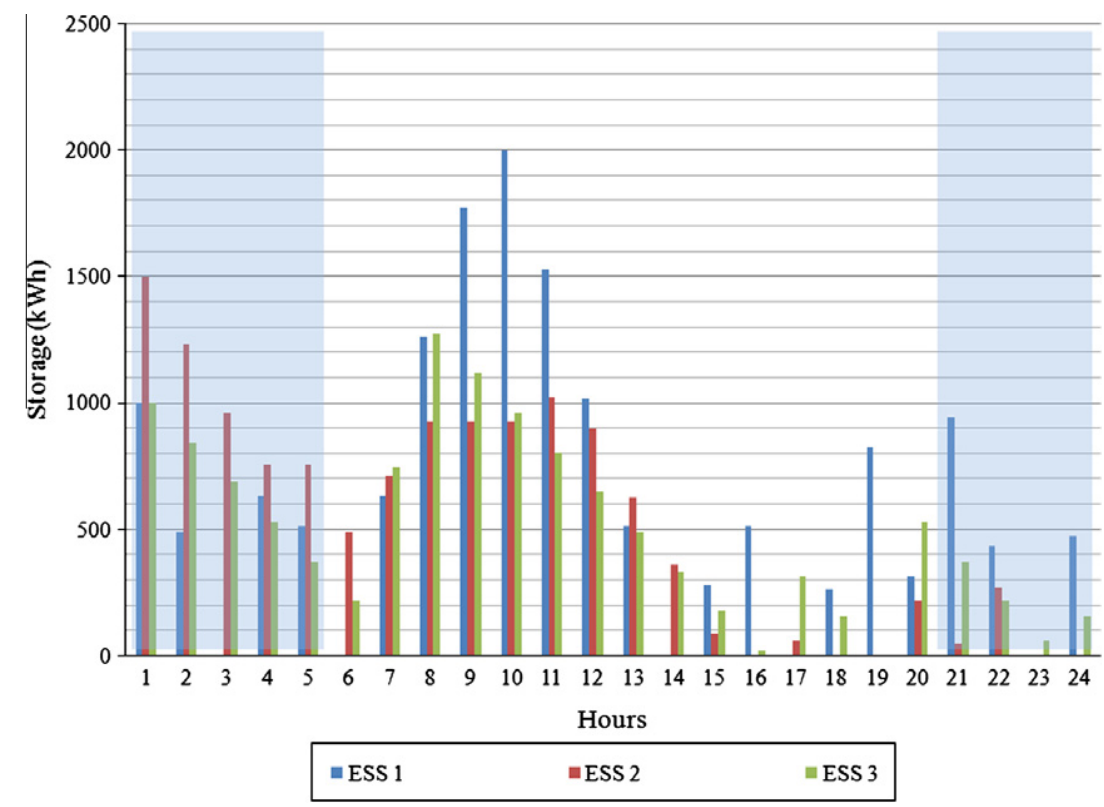

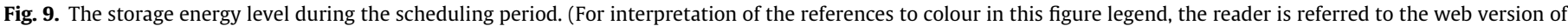
this article.)

in providing energy, these units mostly prefer to discharge and assist the grid in not asking for large amounts of energy. It can be clearly seen in Fig. 9 that the ESSs do not charge significantly during the hours that the solar unit is unavailable.

\section{Conclusions and future work}

Most of the models introduced in the literature define the generation scheduling plan as a single step procedure, without considering the need for data exchange between the producers and the system operator. However, the methods that were based on coordination between the members lacked the security consideration, the main concern of the operators. Bilateral data exchange, the technical basis for the coordination procedure, is available in smart grids. In the proposed method the DSO solves the hourly unit commitment, considering the AC power flow security constraints and reliability issues. In essence, the proposed method tries to introduce effective legislation for maintenance planning in smart grids by clearly defining the main objective of each side.

This multiperiod optimal power flow-based method increases the revenue of the DSO while trying to remember the orientation of producers toward maximum profit by having their units online during the high price periods. Minimizing the total system cost is also the main objective of the operator included in the model.

The possible developments that will be discussed in our future publications are the impact of stochastic factors such as load demand fluctuations, random forced outages of generating units and network lines, system reconfiguration due to the planned and forced outages of the transmission lines. The deviation of generating units from their nominal power factor causes more stress on the system and the units, and consequently affects the maintenance costs. Therefore, this criterion can be included in the future works to introduce a comprehensive outage planning module for short-term generation scheduling.

\section{Acknowledgements}

This work is supported by FEDER Funds through COMPETE program and by National Funds through FCT under the projects
FCOMP-01-0124-FEDER: PEst-OE/EEI/UI0760/2011, PTDC/EEAEEL/099832/2008, and PTDC/SEN-ENR/099844/2008.

\section{References}

[1] Farhangi $\mathrm{H}$. The path of the smart grid. IEEE Power Energy Mag 2010;8(1):18-28.

[2] Conejo JC, García-Bertrand R, Díaz-Salazar M. Generation maintenance scheduling in restructured power systems. IEEE Trans Power Syst 2005;20(2):984-92.

[3] Vale Z, Pinto T, Praca I, Morais H. MASCEM: electricity markets simulation with strategic agents. IEEE Intell Syst 2011;26(2):9-17.

[4] Fotouhi MA, Tafreshi SMM. Strategic maintenance scheduling of distributed generations in oligopolistic electricity markets. IEEE Electr Power Energy Conference; 2009.

[5] Fu Y, Shaidehpour M, Li Z. Security-constrained optimal coordination of generation and transmission maintenance outage scheduling. IEEE Trans Power Syst 2007;22(3):1302-13.

[6] Fu Y, Li Z, Shaidehpour M, Zheng T, Litvinov E. Coordination of midterm outage scheduling with short-term security-constrained unit commitment. IEEE Trans Power Syst 2009;24(4):1818-30.

[7] Fu Y, Shaidehpour M, Li Z. Security-constrained unit commitment with AC constraints. IEEE Trans Power Syst 2005;20(3):1538-50.

[8] Silva MR, Vale Z, Khodr HM, Ramos C, Yusta JM. Optimal dispatch with reactive power compensation by genetic algorithm. IEEE Trans Distrib Conf Exposition 2010:1-7.

[9] Morais H, Kádár P, Faria P, Vale ZA, Khodr HM. Optimal scheduling of a renewable micro-grid in an isolated load area using mixed-integer linear programming. Renew Energy 2010;35(1):151-6.

[10] Ren H, Gao W. A MILP model for integrated plan and evaluation of distributed energy systems. Appl Energy 2010;87(3):1001-14.

[11] Hawkes AD, Leach MA. Modelling high level system design and unit commitment for a microgrid. Appl Energy 2009;86(7-8):1253-65.

[12] Chinese D, Meneghetti A. Optimisation models for decision support in the development of biomass-based industrial district-heating networks in Italy. Appl Energy 2005;82(3):228-54.

[13] Tsikalakis AG, Hatziargyriou ND. Centralized control for optimizing microgrids operation. IEEE Trans Energy Convers 2008;23(1):241-8.

[14] Faria P, Vale Z, Soares J, Ferreira J. Demand response management in power systems using Particle Swarm Optimization approach. IEEE Intelligent Systems (2011), doi:10.1109/MIS.2011.35.

[15] Medina J, Muller N, Roytelman I. Demand response and distribution grid operations: opportunities and challenges. IEEE Trans Smart Grid 2010;1(2):193-8.

[16] Huang Y. Market concepts and regulatory bottlenecks for smart distribution grids in EU countries. MSc thesis, Stockholm, Sweden, KTH Royal Institute of Technology; 2011.

[17] Rau NS, Wan YH. Optimum location of resources in distributed planning. IEEE Trans Power Syst 1994;9(4):2014-20.

[18] Wang C, Nehrir MH. Analytical approaches for optimal placement of distributed generation sources in power systems. IEEE Trans Power Syst 2004;19(4):2068-76. 
[19] Brown RE. Impact of Smart Grid on distribution system design. IEEE Power and Energy Society General Meeting; 2008.

[20] Ipakchi A, Albuyeh F. Grid of the future. IEEE Power Energy Mag 2009;7(2):52-62.

[21] Bruno S, Lamonaca S, Rotondo G, Stecchi U, La Scala M. Unbalanced threephase optimal power flow for smart grids. IEEE Trans Ind Electron 2011;58(10):4504-13.

[22] European Commission. Task Force for Smart Grids. Expert Group 3: Roles and Responsibilities of Actors involved in the Smart Grids Deployment. 2011. <http://ec.europa.eu/energy/gas_electricity/smartgrids/doc/expert_group3. pdf>.

[23] Vaccaro A, Popov M, Villacci D, Terzija V. An integrated framework for smart microgrids modeling, monitoring, control, communication, and verification. Proc IEEE 2011;99(1):119-32.

[24] Korpaas M, Holen AT, Hildrum R. Operation and sizing of energy storage for wind power plants in a market system. Electr Power Energy Syst 2003;25(8):599-606.
[25] Shaidehpour M. Role of security in optimal maintenance scheduling. Second Carnegie Mellon Conf in Electric Power Systems; 2006. p. 1-6.

[26] Eshraghnia R, Shanechi MHM, Mashhadi HR, Karsaz A. A novel approach for maintenance scheduling of generating units in a competitive environment. IEEE Power India Conference; 2006.

[27] Khodr HM, Crespo JM, Matos MA, Pereira J. Distribution systems reconfiguration based on OPF using Benders decomposition. IEEE Trans Power Delivery 2009;24(4):2166-76.

[28] Brooke A, Kendrick D, Meeraus A, Raman R. GAMS: a user's guide. GAMS Development Corporation, Washington, DC; 2008.

[29] GAMS Development Corporation, Washington, DC 20007, USA. GAMS - the solver manuals, GAMS user notes; January 2001. 